\title{
Decreased biofilm formation in Proteus mirabilis after short-term exposure to a simulated microgravity environment
}

\author{
Dapeng Wang $^{1}$ (D) $\cdot$ Po Bai $^{2}$ (D) $\cdot{\text { Bin } \text { Zhang }^{3} \text { (D) } \cdot \text { Xiaolei Su }}^{1} \cdot$ Xuege Jiang ${ }^{1} \cdot$ Tingzheng Fang $^{1} \cdot$ Junfeng Wang ${ }^{1,4}$ (D) \\ Changting Liu ${ }^{1,4}$
}

Received: 10 April 2021 / Accepted: 3 August 2021 / Published online: 23 September 2021

(C) The Author(s) 2021

\begin{abstract}
Background Microbes threaten human health in space exploration. Studies have shown that Proteus mirabilis has been found in human space habitats. In addition, the biological characteristics of $P$. mirabilis in space have been studied unconditionally. The simulated microgravity environment provides a platform for understanding the changes in the biological characteristics of $P$. mirabilis. Objective This study intends to explore the effect of simulated microgravity on P. mirabilis, the formation of $P$. mirabilis biofilm, and its related mechanism.

Methods The strange deformable rods were cultured continuously for 14 days under microgravity simulated in high-aspect rotating vessels (HARVs). The morphology, growth rate, metabolism, and biofilm formation of the strain were measured, and the phenotypic changes of $P$. mirabilis were evaluated. Transcriptome sequencing was used to detect differentially expressed genes under simulated microgravity and compared with phenotype.

Results The growth rate, metabolic ability, and biofilm forming ability of $P$. mirabilis were lower than those of normal gravity culture under the condition of simulated microgravity. Further analysis showed that the decrease of growth rate, metabolic ability, and biofilm forming ability may be caused by the downregulation of related genes ( $p s t S$, sodB, and fumC).

Conclusion The simulated microgravity condition enables us to explore the potential relationship between bacterial phenotype and molecular biology, thus opening up a suitable and constructive method for medical fields that have not been explored before. It provides a certain strategy for the treatment of $P$. mirabilis infectious diseases in space environment by exploring the microgravity of P. mirabilis.
\end{abstract}

Keywords Simulated microgravity $\cdot$ Proteus mirabilis $\cdot$ High-aspect rotating vessel $\cdot$ Biofilm formation $\cdot$ Transcriptome sequencing

\section{Introduction}

Manned space technology is developing rapidly. Astronauts and cabin equipment inevitably bring microbes, including

Dapeng Wang, Po Bai and Bin Zhang contributed equally to this work.

Responsible Editor: Jorge Luiz Mello Sampaio.

Junfeng Wang

wangjf72@hotmail.com

Changting Liu

changtingliu1202@sohu.com

1 Department of Respiratory and Critical Care Medicine, The Second Medical Center \& National Clinical Research Center for Geriatric Disease, Medical School of Chinese PLA, Beijing, People's Republic of China bacteria, fungi, and viruses, into space in the process of manned spaceflight. The space environment is characterized by a series of prominent features more than $100 \mathrm{~km}$ above the earth's surface, including strong ionizing radiation, high
2 Respiratory Diseases Department, PLA Rocket Force Characteristic Medical Center, Beijing, People's Republic of China

3 Respiratory Diseases Department and Critical Care Medicine, Binzhou Medical University Hospital, Binzhou, People's Republic of China

4 Respiratory Diseases Department, The Second Medical Center of PLA General Hospital, Beijing 100853, People's Republic of China 
vacuum, ultra-low temperature, ultra-high temperature, and weightlessness [1-3]. Various factors in the space environment can induce genetic changes of microorganisms, and then affect the phenotypic characteristics of microorganisms, including morphology, growth rate, biofilm forming ability, virulence, and drug resistance, which may induce infectious diseases and affect the health of resident personnel. As a consequence, it is of great significance to study the phenotypic characteristics of microorganisms in space environment.

Proteus mirabilis belongs to Proteus and widely exists in soil, water, and feces [4]. In addition, P. mirabilis is highly pathogenic and is one of the main causes of persistent and refractory urinary tract infections. Studies have shown that $P$. mirabilis has been tested for in space and post-flight astronaut samples in human habitats $[5,6]$. The occurrence of infectious diseases is more likely to occur due to the decline of human immunity in the space environment. As a consequence, how to prevent and treat the infection caused by P. mirabilis in the space environment is worthy of attention. However, studies on the biological characteristics of $P$. mirabilis in the space environment have not been carried out due to concerns about the cost, time, and safety of spacecraft flight.

Microgravity environment refers to the environment in which the apparent weight of the system is much smaller than its actual weight under the action of gravity. In fact, it is the most important environmental factor encountered in the process of manned spaceflight. Microgravity simulation experiment has become an important and necessary preparation for the development of manned spaceflight. The biological characteristics of microorganisms under simulated microgravity have been widely studied in recent years. Some results show that under the simulated microgravity conditions, microorganisms show the same biological characteristics as the space environment. For example, it was found that the virulence of Salmonella typhimurium increased under simulated microgravity, which was consistent with that of Salmonella typhimurium under space microgravity. Further studies at the molecular level also show that RNA-binding protein Hfq is involved in the process of simulating the effects of microgravity and space environment. A simulated microgravimeter was used to observe the changes in biological characteristics and internal mechanism of $P$. mirabilis under microgravity. It can provide a strategy for the prevention and treatment of infectious diseases caused by $P$. mirabilis in space environment in the future.

\section{Materials and methods}

\section{Bacterial strains and culture conditions}

The whole study used $P$. mirabilis clinically separated from the urine of a male patient. The API20NE identification system (BioMèrieux, Craponne, France) and 16S rDNA gene sequencing were applied to identify the strains. The $P$. mirabilis strains grew aerobically in Luria-Bertani (LB) medium (with $0.5 \%$ agar) at $37{ }^{\circ} \mathrm{C}$. The experimental (simulated microgravity, SMG) and the normal gravity (NG) control were set up in a high-aspect rotating vessel (HARV) bioreactor (Synthecon, Inc., Houston, TX, USA) and cultured continuously. Supplementary Figure 1 shows SMG culturing by rotating the bioreactor with the axis perpendicular to gravity, while the cultivation under NG is realized in case of its axis parallel to gravity [7]. P. mirabilis was inoculated in the HARV bioreactor with a dilution of 1:200. Each bioreactor was fully filled with fresh LB medium of $\sim 55 \mathrm{ml}$, and the bubbles were removed carefully. After culturing in HARVs at $37{ }^{\circ} \mathrm{C}$ for $24 \mathrm{~h}$ at $25 \mathrm{rpm}$, the $P$. mirabilis cultures under SMG and NG were diluted and transferred into another HARVs fully filled with LB medium. P. mirabilis inoculation was cultured in the HARV bioreactors for 2 weeks. $P$. mirabilis cultured under SMG was called PMML strain, while that cultured under NG was called PMGL strain. The PMML and PMGL groups were serially diluted in phosphatebuffered saline (PBS), plated on LB agar, and counted. The cultures obtained were tested in various ways.

\section{Phenotypic analysis}

\section{Scanning electron microscopy (SEM)}

PMML and PMGL strains were cultured in LB medium, washed with sterile phosphate-buffered saline (PBS; $\mathrm{pH}$ 7.4), and fixed with $4 \%$ glutaraldehyde overnight. The samples were washed again with PBS, dehydrated in increasing grades of ethanol, and then critical pointdried. After coating the specimens with gold-palladium, they were observed using an FEI Quanta 200 scanning electron microscope (USA).

\section{Growth rate assay}

The Bioscreen C system and BioLink software (Lab Systems) were applied to monitor $P$. mirabilis growth. The overnight growing of $P$. mirabilis strains was made in LB medium at 37 ${ }^{\circ} \mathrm{C}$. Twenty microliters of the overnight culture at a concentration of $10^{6} \mathrm{CFU} / \mathrm{ml}$ was cultivated in Bioscreen C 96-well microtiter plates (Lab Systems). The plates containing fresh LB growth medium of $350 \mu \mathrm{l}$ per well were incubated under continuous shaking at the maximum amplitude for $24 \mathrm{~h}$ at 37 ${ }^{\circ} \mathrm{C}$. The $\mathrm{OD}_{630}$ was measured every 2 h. P. mirabilis growth experiments were performed in triplicate.

\section{Carbon source utilization and chemical sensitivity assay}

The biochemical features of two strains were tested by using the Biolog GENIII MicroPlate (Biolog, CA) and 94 
phenotypic tests, including utilization of 71 carbon sources and assays of 23 chemical sensitivity. The overnight growing of $P$. mirabilis strains on agar plates was made at $37^{\circ} \mathrm{C}$. The centrifugal tube containing two strains was washed with PBS and inoculated into the IF-A inoculum (Biolog, CA). A turbidimeter was used to adjust the concentration of $P$. mirabilis suspension to $10^{8} \mathrm{CFU} / \mathrm{ml}$, and the suspension of $100 \mu \mathrm{l}$ was inoculated into each well of the 96-well plate. After 24-h incubation at $37^{\circ} \mathrm{C}$, an automated Biolog microplate reader at $630 \mathrm{~nm}$ was used to measure the absorbance. The experiment was carried out in triplicate.

\section{Biofilm assay}

Crystal violet staining (a) After being diluted (1:100) in 5-ml LB medium, the PMML and PMGL cultures were moved to glass tubes and incubated at $37^{\circ} \mathrm{C}$ for $24 \mathrm{~h}$ at $200 \mathrm{rpm}$. The planktonic bacteria were removed. Soon afterwards, deionized water was used to wash each tube three or four times. Then, $0.1 \%$ of crystal violet dye was used to stain the glass tubes for $15 \mathrm{~min}$ at $37^{\circ} \mathrm{C}$. The experiment was carried out in triplicate.

Crystal violet staining (b) After adding approximately $200 \mu \mathrm{l}$ of the $P$. mirabilis suspension culture $\left(1-5 \times 10^{7} \mathrm{CFU} / \mathrm{ml}\right)$ to 96-well microculture plates, the mixture was incubated at 37 ${ }^{\circ} \mathrm{C}$ for $24 \mathrm{~h}$. PBS was used to wash the wells two times. In total, $200 \mu \mathrm{l}$ of $0.1 \%$ crystal violet was employed to stain the cells (Sigma, St. Louis, MO, USA) for $30 \mathrm{~min}$ and washed with PBS. Next, the microculture plates were dried, and the dissolution of the stained biomass samples in $95 \%$ ethanol was made. The Thermo Multiskan Ascent Instrument (Thermo, USA) was applied to determine $\mathrm{OD}_{570}$ for each well. The experiments were carried out in triplicate.

Analysis of biofilm formation ability via confocal laser scanning microscopy (CLSM) The two strains were cultured on LB medium overnight and inoculated in 35-mm confocal dishes (Solarbio, Beijing, China). The plates were incubated at $37^{\circ} \mathrm{C}$ for $24 \mathrm{~h}$ and stained with a Filmtracer LIVE/DEAD Biofilm Viability Kit (Invitrogen, Carlsbad, CA, USA) in accordance with the manufacturer's instructions. Three image stacks were obtained from each sample randomly by CLSM using a Leica TCS SP8 microscope with a Lecia TCS SP8 CSU imaging system. All the experiments were carried out in triplicate.

\section{Transcriptomic sequencing and comparison}

Sequencing and filtering $P$. mirabilis cells were collected after 5 -min centrifugation at $8000 \times g$ at $4{ }^{\circ} \mathrm{C}$. The RNeasy Protect Bacteria Mini Kit (Qiagen, Germany) was used to extract total RNA from the two strains according to the manufacturer's instructions. The 10-min centrifugation of $P$. mirabilis was made at $10,000 \times g$ at $4{ }^{\circ} \mathrm{C}$. Chloroform added with the supernatant was mixed for $15 \mathrm{~s}$. After being transferred into a tube filled with isopropanol, the upper aqueous phase was centrifuged at $13,600 \times \mathrm{g}$ for $20 \mathrm{~min}$ at $4{ }^{\circ} \mathrm{C}$. After removing the supernatant, 3-min centrifugation of the mixture of precipitate and ethanol was made at $12,000 \times g$ and $4{ }^{\circ} \mathrm{C}$. Soon afterwards, the supernatant was discarded, and the 20-s centrifugation of the sample was made at $12,000 \times g$ and $4{ }^{\circ} \mathrm{C}$. After removing the residual liquid by air-drying, the dissolution of RNA pellet in RNase-free water was made. The purity of the samples is tested by NanoDrop ${ }^{\mathrm{TM}}$. Divalent cations were used to fragment the purified mRNAs into small pieces ( $\sim 200 \mathrm{bp})$. The first strand of cDNA using reverse transcriptase and random primers was generated by applying RNA fragments. Following this, DNA polymerase I and RNase $\mathrm{H}$ were used to create the second strand of cDNA. The enrichment and quantification of cDNA fragments were made by using PCR amplification and Qubit 2.0, respectively. Lastly, the BGISEQ-500 was used to construct and sequence cDNA libraries. The SOAPnuke software (version 1.5.2) was applied to filter raw reads, and adapter reads and poly $\mathrm{N}$ reads were removed from the raw data to acquire clean reads. Clean reads were located to the reference genome by Bowtie2-2.2.3 [8]. Gene expression was quantified by HTSeq v0.6.1, and the calculation of the fragments per kilobase of transcript per million mapped reads (FPKM) of each gene was made according to the gene length. Lastly, the mapping of read counts to the genes was made. The DESeq R package (1.18.0) was employed to make differential gene expression analysis of the two strains. Through DESeq analysis, it is considered that genes yielding a $p$ value of $<0.05$ are differentially expressed. The KEGGseq R package was applied to analyze Kyoto Encyclopedia of Genes and Genomes (KEGG) enrichment of differentially expressed genes (DEGs). It was considered that DEGs with a $p$ value of $<0.05$ are statistically significant. KOBAS software was used to assess the statistical enrichment of DEGs in KEGG pathways.

Statistical analysis of gene expression values and DEGs After mapping the clean reads to the reference strain by applying HISAT (version 2.0.4) and Bowtie2 (version 2.2.5) [9], the gene expression level with RSEM [8] was calculated. Cluster software (version 3.0) was applied to make cluster analysis of gene expression, and Java TreeView (3.0) [10] was used to visualize the results of cluster analysis.

Functional annotation and enrichment analysis The genes from the reference sequences in the KEGG database were selected using the BLAST software to compare and annotate gene function. The gene expression profiles of different sample groups were analyzed using cluster analysis. The transcriptome data was analyzed by identifying DEGs according to the KEGG pathway. Gene enrichment analysis was 
performed using KEGG functional annotation. A $p$ value of $\leq$ 0.05 indicated significant enrichment of DEGs.

\section{Quantitative real-time PCR (qPCR)}

Total RNA and random hexamer primers with SuperscriptIII reverse transcriptase (Invitrogen) using qPCR were employed to synthesize cDNA. The StepOnePLUS PCR system (Thermo, USA) with cDNA as the template was used to carry out the experiment in duplicate for each RNA sample. The related fold change of the target genes in the test and controlled RNA samples was determined. The $16 \mathrm{~S}$ rRNA gene was regarded as an internal reference. The primer sequences used in this study are shown in Table 1.

\section{Statistical analysis}

The quantitative experiments were performed in triplicate and the data were represented as the mean \pm standard deviation (S.D.). A statistical comparison of the data was conducted using two-tailed Student's $t$-test. Statistical significance between the two groups was defined as $p \leq 0.05$.

\section{Results}

\section{Phenotypic characteristics}

\section{Electron microscopy findings}

Scanning electron microscopy was performed to observe single-cell morphology in the PMML and PMGL strains. The results showed that the PMML had no changes in

Table 1 Primers for the target genes used in qRT-PCR

\begin{tabular}{|c|c|c|c|}
\hline Gene name & $\begin{array}{l}\text { Size } \\
\text { (bp) }\end{array}$ & & Primer sequences ( $5 \mathrm{q}$ to $\left.3^{\prime}\right)$ \\
\hline \multirow[t]{2}{*}{$16 \mathrm{~S}$} & \multirow[t]{2}{*}{104} & Forward & $\begin{array}{l}\text { TCACCTAGGCGACG } \\
\text { ATCTCT }\end{array}$ \\
\hline & & Reverse & $\begin{array}{l}\text { GTGCAATATTCCCC } \\
\text { ACTGCT }\end{array}$ \\
\hline \multirow[t]{2}{*}{ PMI_RS06255 } & \multirow[t]{2}{*}{111} & Forward & $\begin{array}{l}\text { GAACCAAACCGTGA } \\
\text { GCGTAT }\end{array}$ \\
\hline & & Reverse & $\begin{array}{l}\text { AGCTTTCTTCGCGA } \\
\text { TCTCTG }\end{array}$ \\
\hline \multirow[t]{2}{*}{ PMI_RS06750 } & \multirow[t]{2}{*}{94} & Forward & $\begin{array}{l}\text { TGGTTCAGGTTGGA } \\
\text { CTTGGT }\end{array}$ \\
\hline & & Reverse & $\begin{array}{l}\text { ATCTTCGCCAGAAA } \\
\text { CAGGTG }\end{array}$ \\
\hline PMI_RS12550 & 105 & Forward & $\begin{array}{l}\text { CAGGTTAGCTCCGG } \\
\text { AAAGTG }\end{array}$ \\
\hline
\end{tabular}

intercellular mucus and had smooth cell walls than the PMGL strains (Fig. 1).

\section{Growth rate assay}

A slight difference was observed between the two strains in terms of growth rate with time (Fig. 2, Table 2). When compared with PMGL, the PMML strain exhibited a decreased growth rate, especially after $14 \mathrm{~h}(p=0.0095)$.

\section{Chemical sensitivity and carbon source utilization assays}

The chemical sensitivity assay showed decreased utilization of $\alpha$-D-lactose and D-mannose $(p=0.0226$ and $p=0.046$, respectively) by PMML compared to that by PMGL (Fig. 3). It is speculated that $P$. mirabilis adapts to a new environment by changing the characteristics of its metabolism, as seen in the simulated microgravity environment. However, the mechanisms involved in this metabolic change are still unclear. Carbon source utilization assays showed that there were no significant differences between other metabolites and stress response pores. However, the Biolog experiment of this study was completed on ground, and not in an actual space environment and, therefore, requires further exploration.

\section{Biofilm assay}

The biofilm forming ability of $P$. mirabilis The biofilm forming ability of $P$. mirabilis is considered a significant phenotype. This ability was analyzed in the PMML and PMGL cultures after a 2 -week cultivation. The biofilms formed on the glass tubes were monitored by crystal violet staining (a). $\mathrm{OD}_{570}$ was measured after crystal violet staining (b). The adhered pellicles were further quantified using the $\mathrm{OD}_{570}$ values. PMGL showed a higher biofilm forming ability than PMML $(p=0.0022)$ (Fig. 4).

Analysis of biofilm formation ability of $P$. mirabilis strain using CLSM Biofilms of PMML and PMGL strains were cultured in 35-mm confocal dishes. Cells were stained with a Filmtracer LIVE/DEAD Biofilm Viability Kit, and biofilm formation ability was tested using confocal laser scanning microscopy (CLSM). Green fluorescence indicates live cells; red fluorescence indicates dead cells. "***” represents adjusted $p$ value $<0.01$; “*”represents adjusted $p$ value $<0.05$. (a) Analysis of biofilm formation ability of PMML strain using CLSM. (b) Analysis of biofilm formation ability of PMGL strain using CLSM. (c) Analysis of biofilm formation ability of PMML and PMGL strain using CLSM (Fig. 5; Supplementary Figure 2). 


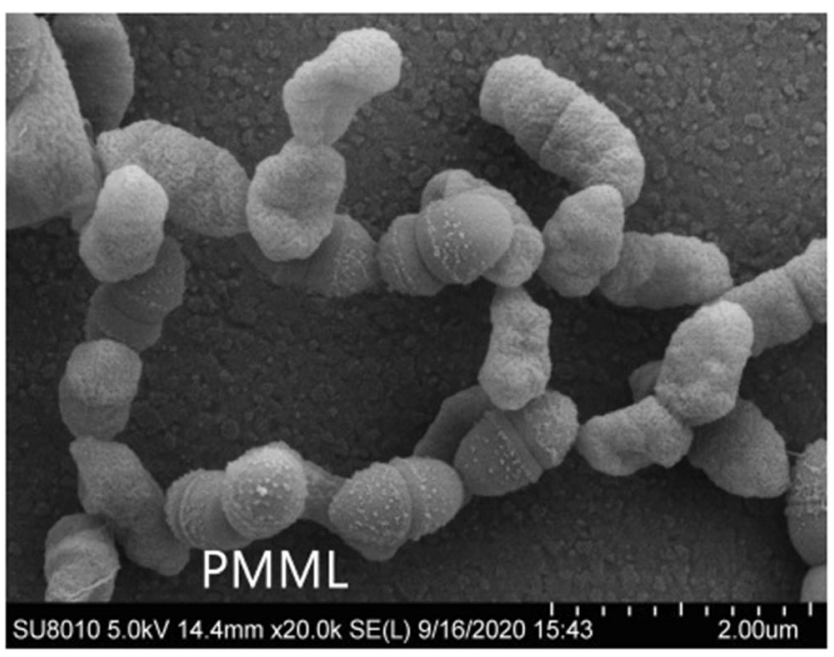

Fig. 1 Experimental setup of the high-aspect rotating vessel bioreactors. $P$. mirabilis cells in the HARV bioreactor are grown under the simulated microgravity (SMG) condition with its axis of rotation perpendicular to

\section{RNA-Seq mapping and comparative genomic analysis}

According to the functional analysis of the KEGG pathway, all DEG clusters were analyzed. Both upregulated and downregulated genes were identified. In PMML, approximately 12 genes were upregulated, and 17 genes were downregulated (Supplementary Figure 3).

We found that the downregulated genes outnumbered the upregulated genes, suggesting that gene metabolism expression was inhibited in PMML. Additionally, most of the DEGs were associated with amino acid metabolism, sugar metabolism, transport, and signal transduction (Supplementary Figure 4; Table 3). Three DEGs related to biofilm metabolism were identified in PMML, including PMI_RS12550 (pstS), PMI RS06750 $(\operatorname{sod} B)$, and PMI RS06255 (fumC). The above results were verified using qPCR.

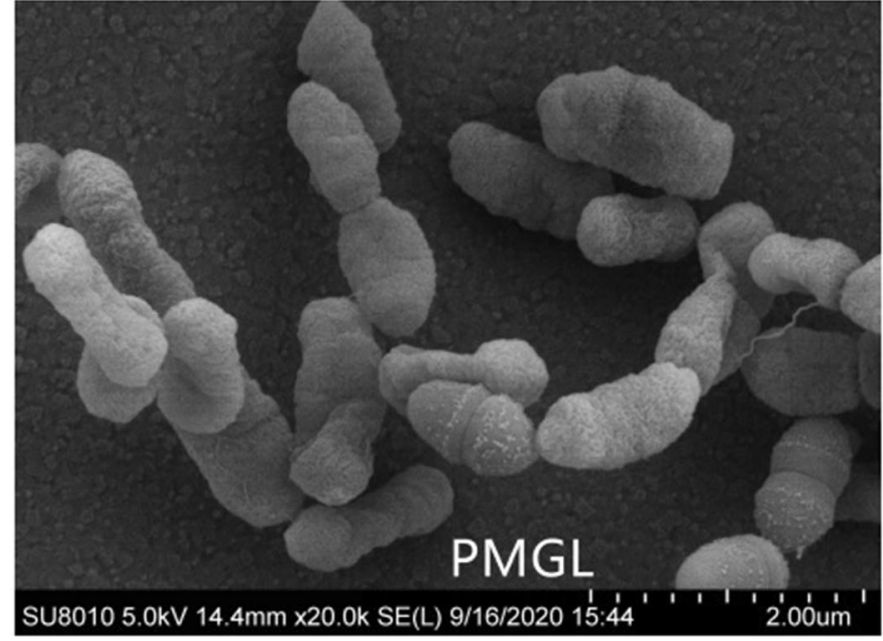

gravity or grown under the normal gravity $(\mathrm{NG})$ with its axis of rotation vertical to gravity. The bioreactors are filled with LB medium and the bubbles are removed

\section{mRNA expression levels of key genes related to biofilm formation}

The mRNA expression levels of key genes related to biofilm formation were analyzed to determine the mechanisms of biofilm formation by PMML and PMGL. PMML exhibited decreased $p s t S$, sodB, and fumC levels compared to PMGL ( $p=$ $0.025, p=0.0056$, and $p=0.004$, respectively) (Fig. 6).

\section{Discussion}

P. mirabilis was exposed to simulated microgravity for 14 days using HARV. In addition, its phenotypic characteristics, including morphology, growth rate, metabolism, and biofilm formation ability, were analyzed. P. mirabilis cultured under
Fig. 2 Scanning electron micrographs (SEM) of PMML and PMGL strains. a SEM of the PMML strain. b SEM of the GS1 strain

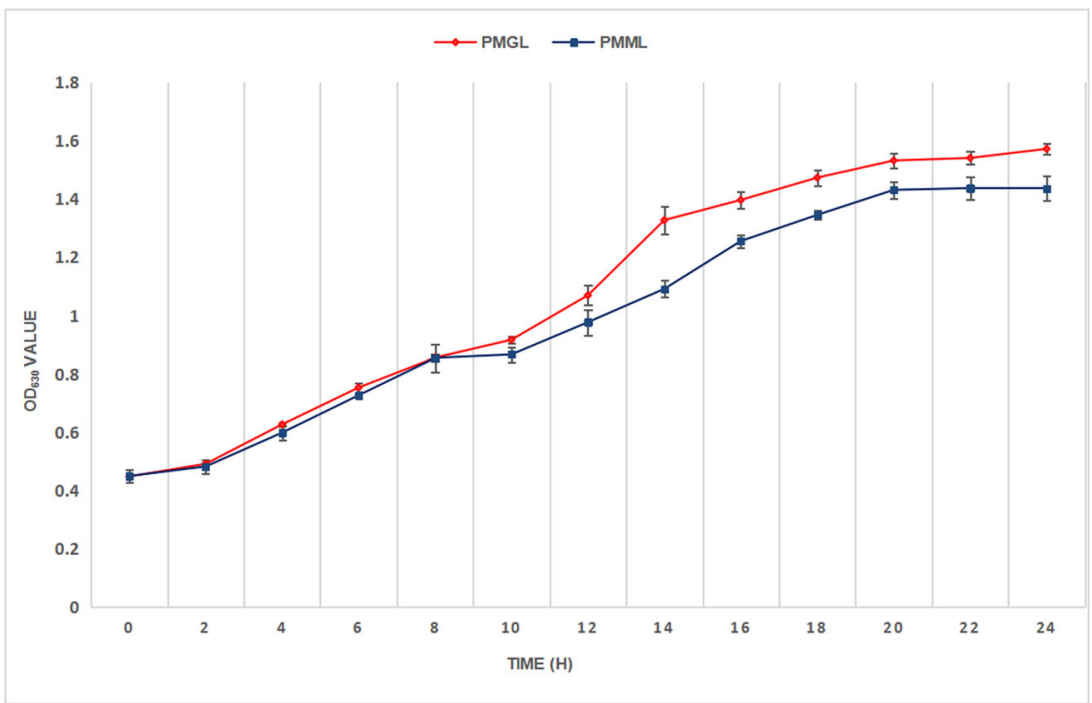


Table $2 \mathrm{OD}_{630}$ values of PMML and PMGL

\begin{tabular}{llll}
\hline Time (h) & PMML & PMGL & $p$ value \\
\hline 0 & $0.4497 \pm 0.0222$ & $0.4480 \pm 0.0066$ & 0.873 \\
2 & $0.4823 \pm 0.0232$ & $0.4913 \pm 0.0047$ & 0.627 \\
4 & $0.5987 \pm 0.0264$ & $0.6270 \pm 0.0062$ & 0.251 \\
6 & $0.7270 \pm 0.0066$ & $0.7537 \pm 0.0168$ & 0.172 \\
8 & $0.7780 \pm 0.0476$ & $0.8553 \pm 0.0110$ & 0.183 \\
10 & $0.8663 \pm 0.0262$ & $0.9167 \pm 0.0131$ & 0.155 \\
12 & $0.9770 \pm 0.0439$ & $1.0697 \pm 0.0343$ & 0.018 \\
14 & $1.0917 \pm 0.0280$ & $1.3280 \pm 0.0485$ & 0.031 \\
16 & $1.2560+0.0216$ & $1.3973 \pm 0.0279$ & 0.010 \\
18 & $1.346 \pm 0.0147$ & $1.4740 \pm 0.0269$ & 0.026 \\
20 & $1.4320 \pm 0.0293$ & $1.5330 \pm 0.0246$ & 0.021 \\
22 & $1.4380 \pm 0.0397$ & $1.5417 \pm 0.0216$ & 0.024 \\
24 & $1.4370 \pm 0.0416$ & $1.5730 \pm 0.0180$ & 0.023 \\
\hline
\end{tabular}

the condition of SMG showed a decrease in growth rate, metabolic ability, and biofilm formation ability. Further analysis showed that the decrease of biofilm forming ability may be related to the downregulated expression of genes related to biofilm formation ( $p s t S, \operatorname{sodB}$, and fumC). It is the first study of $P$. mirabilis in a SMG environment, providing a certain basis for the prevention and treatment of $P$. mirabilis infectious diseases in the future space environment.

Compared with PMGL, the proliferation of PMML was significantly slower than that of PMGL in the logarithmic growth phase. In addition, the growth rate of PMML decreased, which may be related to the downregulation of the genes involved and changes in metabolism and ribosomal structure. Previous studies have also observed changes in the same rate of growth [11]. In addition, Nicholson et al. [12] analyzed the growth rate of Bacillus subtilis spores on the $\mathrm{O} /$ OREOS spacecraft. The flying group of $B$. subtilis cells also showed a lower growth rate compared with the ground control experiment.

The regulation of bacterial metabolism may involve many aspects, including changes in growth rate, utilization of nutrients, and changes in metabolites in a complex environment. Studies have shown that $\alpha$-D-lactose and D-mannose provide necessary energy support in the process of biofilm formation [13-16]. In this study, it was found that in the simulated microgravity environment, the utilization rate of two carbon sources ( $\alpha$-D-lactose and D-mannose) decreased. However, the decrease in the utilization rate of $\alpha$-D-lactose and D-mannose may slow down the biofilm formation or reduce the intensity of biofilm formation. As a result, it further affected the biofilm formation and reduced the growth rate of P. mirabilis.

The biofilm formation in bacteria was first carried out under the action of microgravity in 2001 [17]. Three genes related to biofilm formation, including $p s t S, \operatorname{sodB}$, and fum $C$, were downregulated in SMG. In theory, pst $S$ is a factor related to the ability of bacteria to form biofilm [18]. Some people think that the formation of bacterial biofilm may be induced by $\operatorname{sodB}[19]$. Gabryszewski et al. confirmed the existence of fumC in biofilm and its ability to promote biofilm formation [20]. It has been found that $p s t S, \operatorname{sodB}$, and fumC may affect the growth rate of bacteria [21-23]. Studies have shown that the reduction of biofilm formation will affect the viability of bacteria and reduce the growth rate of bacteria [21]. A study reports that $p s t S$ is involved in the formation of biofilms [24]. The main genes involved in the formation of bacterial biofilm may be $p s t S[25,26]$. We believe that due to the stimulation of microgravity, the expression of $p s t S$ is downregulated, the biofilm forming ability is decreased, and the biofilm becomes thinner, which may affect the growth rate of $P$. mirabilis. Hassett et al. observed that $\operatorname{sod} B$ significantly affected the growth rate of Pseudomonas aeruginosa [22]. SodB is a factor related to biofilm formation, and the decrease of $\operatorname{sodB}$ expression will reduce the biofilm forming ability of bacteria [27]. In $P$. mirabilis, the environmental changes that inhibit the activity of $\operatorname{sod} B$ also lead to the weakening of biofilm, which may reduce the growth rate of $P$. mirabilis. Studies have shown that the growth rate of bacteria is
Fig. 3 Growth curves of two P. mirabilis strains. Growth curves of PMML (blue) and PMGL (red) were determined by measuring the $\mathrm{OD}_{630}$ value, which represents the bacterial concentration. The $\mathrm{OD}_{630}$ value was measured every $2 \mathrm{~h}$ for $24 \mathrm{~h}$
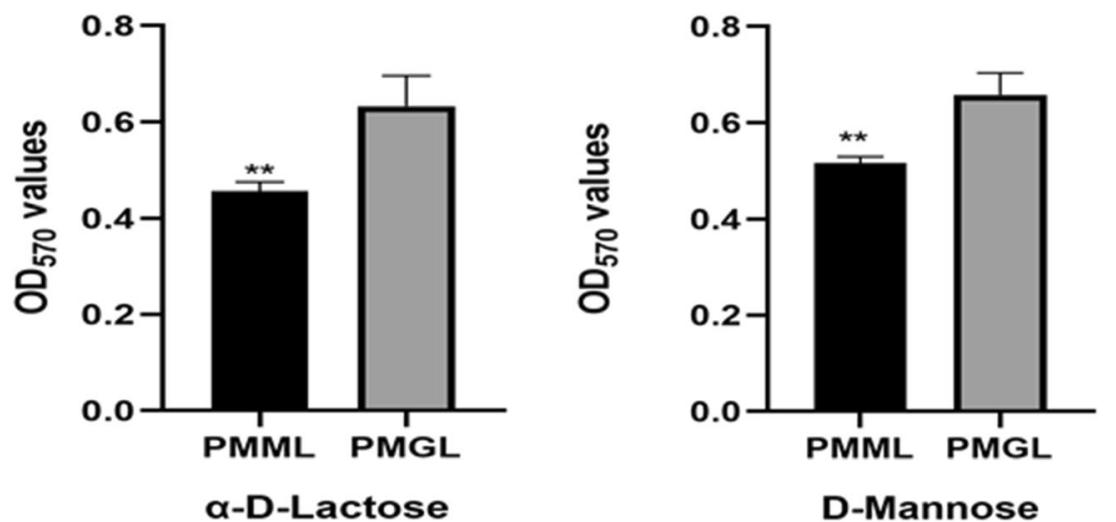
Fig. 4 Chemical sensitivity assays of the two strains. The PMML and PMGL strains were incubated in a Biolog GENIII MicroPlate at $37{ }^{\circ} \mathrm{C}$ for $24 \mathrm{~h}$ in LB medium and tested for carbon source utilization and chemical sensitivity. The effect of simulated microgravity (SMG) on the expression of $\alpha$-D-lactose (a) and D-mannose (b) is shown. Asterisk indicates significant difference at $p<0.05$ (a): $\quad$ Biofilm

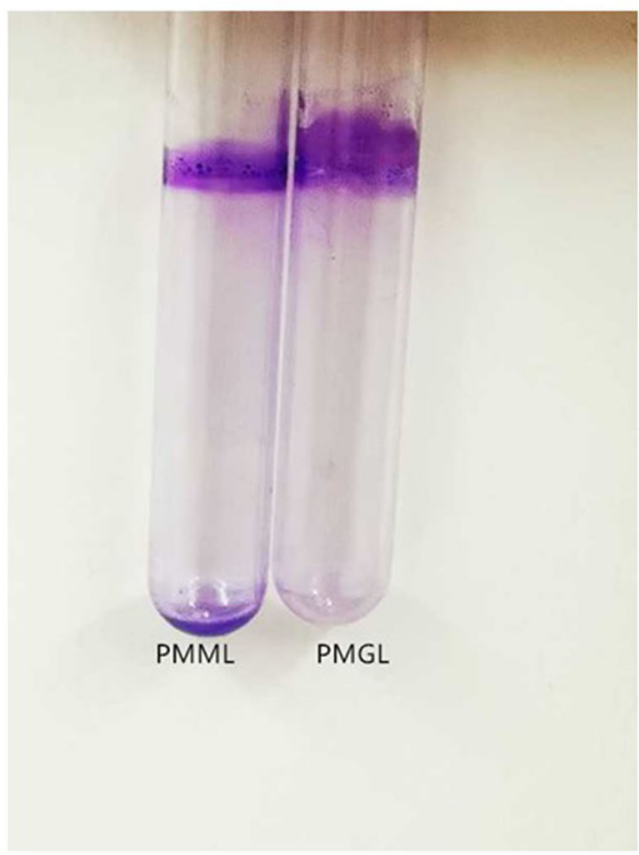

(b): $\quad$ Biofilm

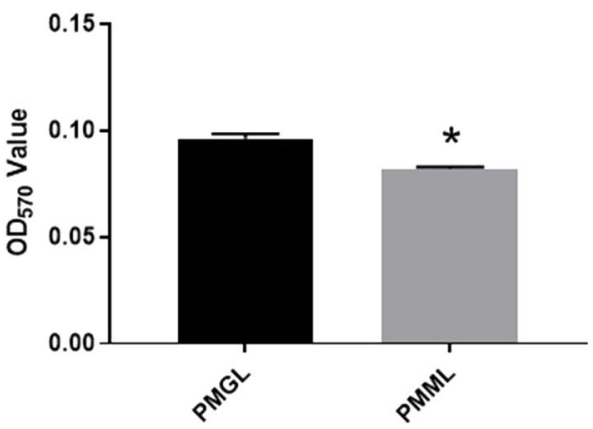

affected by the expression of fumC gene [23]. Fumc is a kind of II enzyme, which is related to the formation of biofilm [28]. In our opinion, the decrease of fum $C$ expression may weaken the formation of biofilm, resulting in a decrease in the growth rate of $P$. mirabilis. As a result, it is speculated that the decrease in the growth rate of $P$. mirabilis under SMG may be attributed to the downregulation of genes related to biofilm formation.

a

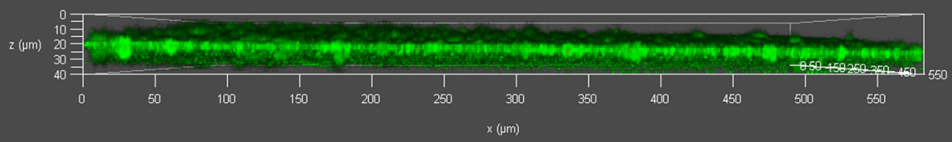

Fig. 5 Analysis of biofilm formation ability via crystal violet staining. The PMML and PMGL strains were cultured in 96-well polystyrene microtiter plates at $37{ }^{\circ} \mathrm{C}$ for $24 \mathrm{~h}$. Biofilm forming ability was measured. The biofilms formed on the glass tubes were analyzed using crystal violet staining (a) and quantified by measuring the absorbance of crystal violet at $570 \mathrm{~nm}$ (b) 


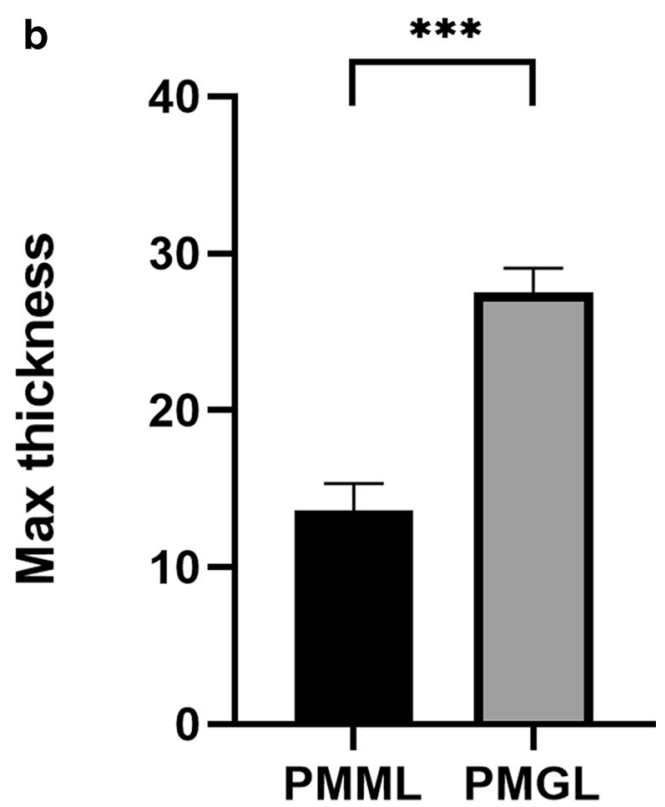

Fig. 5 (continued)
The growth of bacteria on the surface of microorganisms and the ability of biofilm formation under different growth conditions are affected by many factors, including the type of culture, cutting methods, and nutrient utilization. As a result, it showed that Pseudomonas aeruginosa showed increased biofilm formation and new structure formation during spaceflight [29]. In addition, studies have shown that spaceflight reduces the biofilm forming ability of the multidrug-resistant Acinetobacter baumannii [30]. Studies have shown that the uncertainty in the treatment of $P$. mirabilis is closely related to the intensity of biofilm formation [31]. Crystal violet staining and confocal scanning electron microscopy were used to detect the biofilm forming ability of P. mirabilis. Compared with PMGL, the biofilm forming ability of PMML decreased. For further analysis at the transcriptional level, a series of biofilm forming-related genes were detected in the SMG group. As a result, the decrease of biofilm formation ability of $P$. mirabilis in the SMG environment could be attributed to the downregulation of the expression of the above genes.

Table 3 Details of the differentially expressed genes (DEGs)

\begin{tabular}{|c|c|c|c|c|c|c|}
\hline Gene ID & Designation & $\begin{array}{l}\text { Length } \\
\text { (bp) }\end{array}$ & $\begin{array}{l}\text { log2FoldChange } \\
\text { (PMML/PMGL) }\end{array}$ & $\begin{array}{l}\mathrm{Up} / \\
\text { down }\end{array}$ & $p$ value & Gene function \\
\hline PMI_RS14830 & & 2495 & 1.42386006927969 & $\mathrm{Up}$ & $6.96 \mathrm{E}-05$ & Mat/Ecp fimbriae outer membrane usher protein \\
\hline PMI_RS04770 & & 543 & 1.08047931271713 & $\mathrm{Up}$ & 0.00016 & Uridine kinase \\
\hline PMI_RS08220 & & 603 & 1.7635152260287 & Up & $2.50 \mathrm{E}-05$ & $\begin{array}{l}\text { LuxR family transcriptional regulator, } \\
\text { capsular biosynthesis positive transcription } \\
\text { factor }\end{array}$ \\
\hline PMI_RS10390 & mgtE & 1074 & 1.61430565477922 & Up & $3.82 \mathrm{E}-06$ & Fimbrial chaperone protein \\
\hline PMI_RS12550 & & 699 & 1.67510731358992 & Up & $3.82 \mathrm{E}-06$ & Fimbrial chaperone protein \\
\hline PMI_RS09675 & & 861 & 1.84898299118261 & Up & $7.76 \mathrm{E}-05$ & No \\
\hline PMI_RS14835 & & 864 & 1.45666073453672 & Up & $4.40 \mathrm{E}-05$ & Mat/Ecp fimbriae periplasmic chaperone \\
\hline PMI_RS09275 & & 765 & 1.31823468916688 & Up & 0.00043 & Chaperone protein PapD \\
\hline PMI_RS09270 & & 2487 & 1.53135272820167 & Up & $2.42 \mathrm{E}-05$ & Outer membrane usher protein PapC \\
\hline PMI_RS17505 & & & 1.31232107003372 & Up & 0.000109 & $\begin{array}{l}\text { XRE family transcriptional regulator, } \\
\text { master regulator for biofilm formation }\end{array}$ \\
\hline PMI_RS15440 & & 1353 & 1.70558100358398 & Up & 0.00032 & No \\
\hline PMI_RS03790 & pyrD & 1011 & 1.10139831452801 & Up & 8.69E-05 & Type VI secretion system secreted protein Hcp \\
\hline PMI_RS06685 & gstA & 612 & -1.51757779099496 & Down & 0.00028 & Lglutathione S-transferase \\
\hline PMI_RS09365 & $\operatorname{grcA}$ & 384 & -1.7488696118053 & Down & $6.98 \mathrm{E}-05$ & Autonomous glycyl radical cofactor \\
\hline PMI_RS01070 & & 1383 & -1.36339040837153 & Down & $6.35 \mathrm{E}-05$ & Solute carrier family \\
\hline PMI_RS18490 & gpmB & 648 & -1.46649446000024 & Down & $7.08 \mathrm{E}-05$ & Probable phosphoglycerate mutase \\
\hline PMI_RS13405 & & 279 & -1.90346273574243 & Down & 0.00028 & Ethanolamine utilization protein EutM \\
\hline PMI_RS03835 & $\mathrm{rmf}$ & 171 & -1.77234261558484 & Down & 0.00025 & Ribosome modulation factor \\
\hline PMI_RS06255 & fumc & 1398 & -1.53400675009522 & Down & 0.00031 & Fumarate hydratase, class II \\
\hline PMI_RS04910 & & 243 & -1.46880616244531 & Down & 0.00036 & No \\
\hline PMI_RS12610 & & 1071 & -1.35999327826646 & Down & $6.86 \mathrm{E}-06$ & Integrase/recombinase XerC \\
\hline PMI_RS06750 & sodB & 579 & -1.11371491034908 & Down & 9.33E-05 & Superoxide dismutase \\
\hline PMI_RS19020 & $\operatorname{ssr} \mathrm{A}$ & 367 & -2.3513452021666 & Down & 2.07E-06 & No \\
\hline PMI_RS14315 & pstB & 777 & -1.91031855481805 & Down & 0.00043 & Chaperone protein PapD \\
\hline PMI_RS06815 & & 240 & -1.77700473582651 & Down & 0.00015 & Murein lipoprotein \\
\hline PMI_RS05420 & & 519 & -1.5049377019371 & Down & $1.91 \mathrm{E}-05$ & Secretion system secreted protein Hcp \\
\hline PMI_RS05240 & ychH & 279 & -2.48872258916291 & Down & 7.89E-07 & No \\
\hline PMI_RS14300 & pstS & 1041 & -1.88909737452914 & Down & 0.00025 & $\begin{array}{l}\text { Phosphate transport system substrate-binding } \\
\text { protein }\end{array}$ \\
\hline PMI_RS03690 & & 519 & -1.50265019782485 & Down & 8.69E-05 & Type VI secretion system secreted protein Hcp \\
\hline
\end{tabular}


fumc PMI_RS06255

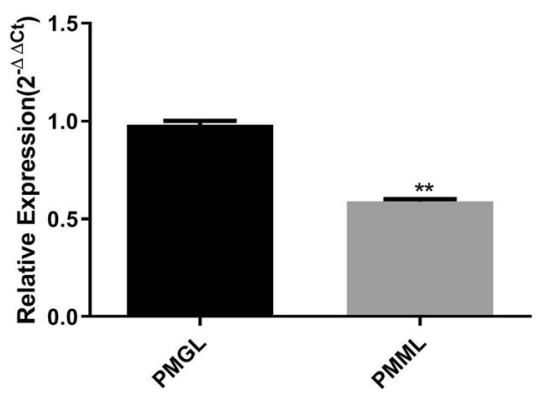

SodB PMI_RS06750

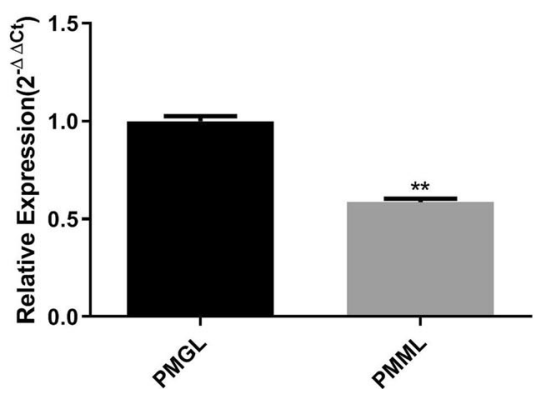

pstS PMI_RS12550

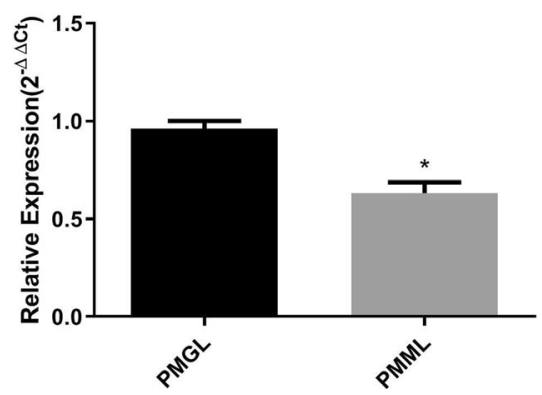

Fig. 6 Analysis of biofilm formation ability via CLSM. a Analysis of biofilm formation ability of PMML strain using CLSM. b Analysis of biofilm formation ability of PMGL strain using CLSM. c Analysis of biofilm formation ability of PMML and PMGL strain using CLSM

\section{Conclusion}

The study provided data showing a decline in growth rate, metabolism, and biofilm formation of P. mirabilis after 14 days of exposure to simulated microgravity. In addition, further analysis showed that the decrease of growth rate, metabolic ability, and biofilm forming ability may be due to the downregulation of biofilm formation and synthesis ( $p s t S$, $\operatorname{sodB}$, and fum C) gene expression. The SMG condition enables us to explore the potential relationship between bacterial phenotype and molecular biology, thus opening up a suitable and constructive method for medical fields that have not been explored before. It provides a certain strategy for the treatment of $P$. mirabilis infectious diseases in space environment by exploring the microgravity of $P$. mirabilis.

Supplementary Information The online version contains supplementary material available at https://doi.org/10.1007/s42770-021-00588-y.

Author contribution Dapeng Wang and Po Bai performed the experiments, analyzed the data, prepared figures and/or tables, and authored or reviewed drafts of the manuscript. Bin Zhang and Xiaolei Su performed the experiments and contributed reagents/materials/analysis tools. Xuege Jiang, Tingzheng Fang, and Li Xu performed the experiments and prepared figures and/or tables. Junfeng Wang conceived and designed the experiments, authored or reviewed drafts of the manuscript, and approved the final manuscript draft. Changting Liu conceived and designed the experiments, authored or reviewed drafts of the manuscript, and approved the final manuscript draft.

Funding This work was supported by the Key Program of Logistics Research (BWS17J030).

\section{Declarations}

Ethics approval The study was approved by the institutional review board (CWO) of the Chinese PLA General Hospital, Beijing, China (ethical number: S2019-327-01).

Conflict of interest The authors declare no competing interests.
Open Access This article is licensed under a Creative Commons Attribution 4.0 International License, which permits use, sharing, adaptation, distribution and reproduction in any medium or format, as long as you give appropriate credit to the original author(s) and the source, provide a link to the Creative Commons licence, and indicate if changes were made. The images or other third party material in this article are included in the article's Creative Commons licence, unless indicated otherwise in a credit line to the material. If material is not included in the article's Creative Commons licence and your intended use is not permitted by statutory regulation or exceeds the permitted use, you will need to obtain permission directly from the copyright holder. To view a copy of this licence, visit http://creativecommons.org/licenses/by/4.0/.

\section{References}

1. Thirsk R, Kuipers A, Mukai C, Williams D (2009) The space-flight environment: the International Space Station and beyond. CMAJ 180(12):1216-1220. https://doi.org/10.1503/cmaj.081125

2. Gerald S (2005) The immune system in space, including Earthbased benefits of space based research. Curr Pharm Biotechnol 6(4):343-349. https://doi.org/10.2174/1389201054553699

3. Song B, Leff LG (2005) Identification and characterization of bacterial isolates from the Mir space station. Microbiol Res 60(2):111117. https://doi.org/10.1016/j.micres

4. Branka B, Nataša F, Vesna EG, Marija K, Krešimir M, Iva Š, Josefa L, Jasmina V, Tomislav M, Gernot Z, Andrea G (2016) Emergence of multidrug-resistant Proteus mirabilis in a long-term care facility in Croatia. Wien Klin Wochenschr 128(11-12):404-413. https:// doi.org/10.1007/s00508-016-1005-x

5. Chaffer CL, Weinberg RA (2011) A perspective on cancer cell metastasis. Science 331:1559-1564. https://doi.org/10.1126/ science. 1203543

6. Castro VA, Thrasher AN, Healy M, Ott CM, Pierson DL (2004) Microbial characterization during the early habitation of the International Space Station. Microb Ecol 47:119-126. https://doi. org/10.1007/s00248-003-1030-y

7. Nickerson CA, Ott CM, Mister SJ, Morrow BJ, Burns-Keliher L, Pierson DL (2000) Microgravity as a novel environmental signal affecting Salmonella enterica serovar Typhimurium virulence. Infect Immun 68(6):3147-3152. https://doi.org/10.1128/iai.68.6. 3147-3152.2000

8. Langmead B, Salzberg SL (2012) Fast gapped-read alignment with Bowtie 2. Nat Methods 9(4):357-359. https://doi.org/10.1038/ nmeth.1923 
9. Li B, Dewey CN (2011) RSEM: accurate transcript quantification from RNA-Seq data with or without a reference genome. BMC Bioinformatics 4(12):323. https://doi.org/10.1186/1471-2105-12323

10. Saldanha AJ (2004) Java Treeview-extensible visualization of microarray data. Bioinformatics 20(17):3246-3248. https://doi.org/ 10.1093/bioinformatics/bth349

11. Su L, Zhou L, Liu J, Cen Z, Wu C, Wang T, Zhou T, Guo T, Fang X, Wang J, Li T, Yin S, Dai S, Zhou P, Zhao J, Fang C, Yang R, Liu C (2014) Phenotypic, genomic, transcriptomic and proteomic changes in Bacillus cereus after a short-term space flight. Adv Space Res 53:18-29. https://doi.org/10.1016/j.asr.2013.08.001

12. Nicholson WL, Ricco AJ, Agasid E, Beasley C, Diazaguado M, Ehrenfreund P, Friedericks C, Ghassemieh S, Henschke M, Hines JW, Kitts C, Luzzi E, Ly D, Mai N, Mancinelli R, McIntyre M, Minelli G, Neumann M, Parra M, Piccini M, Rasay RM, Ricks R, Santos O, Schooley A, Squires D, Timucin L, Yost B, Young A (2011) The O/OREOS mission: first science data from the Space Environment Survivability of Living Organisms (SESLO) payload. Astrobiology 11:951-958. https://doi.org/10.1089/ast.2011.0714

13. Varela JA, Montini N, Scully D, Van der Ploeg R, Oreb M, Boles E, Hirota J, Akada R, Hoshida H, Morrissey JP (2017) Polymorphisms in the LAC12 gene explain lactose utilisation variability in Kluyveromyces marxianus strains. FEMS Yeast Res 17(3):1-13. https://doi.org/10.1093/femsyr/fox021

14. Zhang L, Xu Z, Patel BKC (2007) Bacillus decisifrondis sp. nov., isolated from soil underlying decaying leaf foliage. Int J Syst Evol Microbiol 57(Pt 5):974-978. https://doi.org/10.1099/ijs.0.64440-0

15. Collier WA, De Miranda JC (1955) Bacterien-Haemagglutination. Antonie Van Leeuwenhoek 21:133-140. https://doi.org/10.1007/ bf02543806

16. De Graft-Hanson JA, Heath JL (1990) Effect of D-mannose on motility, presence of flagella, and attachment of Pseudomonas aeruginosa to chicken skin cells. Poult Sci 69(8):1404-1409. https://doi.org/10.3382/ps.0691404

17. McLean RJ, Cassanto JM, Barnes MB, Koo JH (2001) Bacterial biofilm formation under microgravity conditions. FEMS Microbiol Lett 195(2):115-119. https://doi.org/10.1111/j.1574-6968.200

18. Cabral MP, Soares NC, Aranda J, Parreira JR, Rumbo C, Poza M, Valle J, Calamia V, Lasa I, Bou G (2011) Proteomic and functional analyses reveal a unique lifestyle for Acinetobacter baumannii biofilms and a key role for histidine metabolism. J Proteome Res 10(8):3399-3417. https://doi.org/10.1021/pr101299j

19. DePas WH, Hufnagel DA, Lee JS, Blanco LP, Bernstein HC, Fisher ST, James GA, Stewart PS, Chapman MR (2013) Iron induces bimodal population development by Escherichia coli. Proc Natl Acad 110(7):2629-2634. https://doi.org/10.1073/pnas. 1218703110

20. Gabryszewski SJ, Wong Fok Lung T, Annavajhala MK, Tomlinson KL, Riquelme SA, Khan IN, Noguera LP, Wickersham M, Zhao A, Mulenos AM, Peaper D, Koff JL, Uhlemann AC, Prince A (2019) Staphylococcus aureus metabolic adaptation in methicillin-resistant pneumonia. Am J Respir Cell Mol Biol 61(2):85-197. https://doi. org/10.1165/rcmb.2018-0389OC

21. Rogers AH, Pilowsky K, Zilm PS (1984) The effect of growth rate on the adhesion of the oral bacteria Streptococcus mutans and Streptococcus milleri. Arch Oral Biol 29(2):147-150. https://doi. org/10.1016/0003-9969(84)90119-5

22. Hassett DJ, Schweizer HP, Ohman DE (1995) Pseudomonas aeruginosa sodA and sodB mutans defective in manganese- and iron-cofactored superoxide dismutase activity demonstrate the importance of the iron-cofactored form in aerobic metabolism. $\mathrm{J}$ Bacteriol 177(22):6330-6337. https://doi.org/10.1128/jb.177.22. 6330-6337

23. Park SJ, Gunsalus RP (1995) Oxygen, iron, carbon, and superoxide control of the fumarase fumA and fumC genes of Escherichia coli: role of the arcA, fnr, and soxR gene products. Microb Pathog 177(21):6255-6262. https://doi.org/10.1128/jb.177.21

24. O’May GA, Jacobsen SM, Longwell M, Stoodley P, Mobley HLT, Shirtliff ME (2009) The high-affinity phosphate transporter Pst in Proteus mirabilis HI4320 and its importance in biofilm formation. Microbiology 155(Pt 5):1523-1535. https://doi.org/10.1099/mic.0. 026500-0

25. Mudrak B, Tamayo R (2012) The Vibrio cholerae Pst2 phosphate transport system is upregulated in biofilms and contributes to biofilm-induced hyperinfectivity. Infect Immun 80(5):1794-1802. https://doi.org/10.1128/IAI.06277-11

26. Blus-Kadosh I, Zilka A, Yerushalmi G, Banin E (2013) The effect of pstS and phoB on quorum sensing and swarming motility in Pseudomonas aeruginosa. PLoS One 8(9):e74444. https://doi.org/ 10.1371/journal.pone.0074444

27. Chen Y, Wu F, Pang H, Tang J, Cai S, Jian J (2019) Superoxide dismutase B (sodB), an important virulence factor of Vibrio alginolyticus, contributes to antioxidative stress and its potential application for live attenuated vaccine. Fish Shellfish Immunol 89:354-360. https://doi.org/10.1016/j.fsi.2019.03.061

28. Woods SA, Schwartzbach SD, Guest JR (1988) Two biochemically distinct classes of fumarase in Escherichia coli. Biochim Biophys Acta 954(1):14-26. https://doi.org/10.1016/0167-4838(88)90050-7

29. Kim W, Tengra FK, Young Z, Shong J, Marchand N, Chan HK, Pangule RC, Parra M, Dordick JS, Plawsky JL, Collins CH (2013) Spaceflight promotes biofilm formation by Pseudomonas aeruginosa. PLoS One 8(4):e62437. https://doi.org/10.1371/ journal.pone.0062437

30. Zhao X, Yu Y, Zhang X, Huang B, Bai P, Xu C, Li D, Zhang B, Liu C (2019) Decreased biofilm formation ability of Acinetobacter baumannii after spaceflight on China's Shenzhou 11 spacecraft. MicrobiologyOpen 8(6):e00763. https://doi.org/10.1002/mbo3.763

31. Deva AK, Adams WP, Vickery K (2013) The role of bacterial biofilms in device-associate infection. Plast Reconstr Surg 132(5): 1319-1328. https://doi.org/10.1097/PRS.0b013e3182a3c105

Publisher's note Springer Nature remains neutral with regard to jurisdictional claims in published maps and institutional affiliations. 